\title{
REGULAR TYPE OF REAL HYPER-SURFACES IN (ALMOST) COMPLEX MANIFOLDS
}

\author{
JEAN-FRANÇOIS BARRAUD AND EMMANUEL MAZZILLI
}

\begin{abstract}
The regular type of a real hyper-surface $M$ in an (almost) complex manifold at some point $p$ is the maximal contact order at $p$ of $M$ with germs of non singular (pseudo) holomorphic disks. The main purpose of this paper is to give two intrinsic characterizations the type : one in terms of Lie brackets of a complex tangent vector field on $M$, the other in terms of some kind of derivatives of the Levi form.
\end{abstract}

The purpose of this paper is to study order of contact between holomorphic curves (as well pseudoholomorphic curves) and a real hyper-surface. In the integrable case, this invariant is connected to the boundary behavior of the CauchyRiemann equations, the Bergman kernel, invariant metrics, etc.. see for example [5] 3] 4, 9, 8]. One of the difficulty to use this number in function theory on a domain $D$, is due to the fact that in general, it was not known how to calculate it with intrinsic "complex geometry" of the boundary of $D$. In $\mathbb{C}^{2}$ the situation is clear (see [8]); for $\mathbb{C}^{n}(n>2)$, we only know how to compute the order of contact of complex hyper-surface with the natural Lie algebra of the boundary of $D$ (see 2] ). In 2, I. Graham and T. Bloom ask how to characterize the regular "type" in a similar intrinsic way with only one complex vector field; T. Bloom (see 1) succeeds in the pseudoconvex case in $\mathbb{C}^{3}$ but unfortunately, the result is not valid without the pseudoconvexity hypothesis (we think that even with the pseudoconvexity, this result is false in $\mathbb{C}^{n}$ with $n \geq 4$ ).

In this article, we consider an hyper-surface (pseudoconvex or not) in $\mathbb{C}^{n}$ or in $\mathbb{R}^{2 n}$ endowed with an almost complex structure, and characterize its "regular one type", by means of Lie brackets of one "complex tangent vector field" on the hyper-surface (see theorem 1), and by means of derivatives of the "levi form" in some way (see theorem 2). We decided to work in the non-integrable situation, because our main argument shows up naturally in this setting. In particular, we recall the definition of the Levi form in the non-integrable case, show that it has the same properties as the classical one, and use it to define strictly pseudoconvexity and pseudoconvexity.

The plane of the paper is the following: in the first section, we define relevant objects in the non-integrable case and we expound the main results; in section two, we compare jets of holomorphic curves (with contact order $k$ ) with jets of complex tangent vector fields; in section three, we relate the order of contact with complex line sub-bundles of $T^{J}(M)=T M \cap J T M$ (see the definition in the first section) nearly involutive (see theorem 1); in section four, we introduce the "derivatives of the Levi form" and we demonstrate the theorem 2; in the last section, we discuss the base point dependency of the type in (complex) dimension 2 and in a particular case in dimension 3 .

2000 Mathematics Subject Classification. Primary 32T25 ; Secondary 32Q60.

Key words and phrases. finite type, hypersurface, contact order, almost complex, Levi form. 


\section{Notations And MAin RESUlts}

Let us first recall, in the almost complex case, the notions of "complex tangent space", "complex vector fields", "Levi form" and "pseudoconvexity" and derive some of their properties well known in the integrable case.

All along this paper, we will work with the following objects : let $J$ be an almost complex structure on $\mathbb{R}^{2 n}$ (with $J(0)=1$ ) and $M$ an oriented real hyper-surface through 0 in $\mathbb{R}^{2 n}$. Let $T^{J} M=T M \cap J T M$ denote the $J$-invariant part of $T M$. The sections of $T^{J} M$ will be called $J$-tangent or complex tangent vector fields on $M$. We choose a vector field $N$ transversal to $M$ so that we have :

$$
\mathbb{R}^{2 n}=T M \oplus \mathbb{R} N \quad \text { and } T M=T^{J} M \oplus \mathbb{R}(J N) .
$$

Let $\phi: \mathbb{R}^{2 n} \rightarrow \mathbb{R}$ be a defining function for $M$, i.e. a function such that $M=$ $\phi^{-1}(0), 0$ is a regular value of $\phi$, and $\phi$ defines the positive orientation of $M$. Of course, if $\psi$ is another defining function of $M$, then there exists a non negative function $\left.f: \mathbb{R}^{2 n} \rightarrow\right] 0,+\infty[$ such that $\psi=f \phi$. Once a defining function $\phi$ and a metric on $\mathbb{R}^{2 n}$ are chosen, we can take $N$ to be the gradient $\nabla \phi$ of $\phi$ with respect to the metric.

The set $M_{+}=\{\phi>0\}$ will be called the outside, and $M_{-}=\{\phi<0\}$ the inside defined by $M$.

The main purpose of this paper is to characterize the maximal contact order $\Delta_{\text {reg }}^{1}(p)$ of $M$ with regular (eventually pseudo-) holomorphic disks at some point $p$ intrinsically, i.e. in terms of tangent vector fields on $M$. Recall that a germ of map $u:(\mathbb{C}, 0) \rightarrow\left(\mathbb{R}^{2 n}, 0\right)$ is pseudo-holomorphic with respect to $J$, or $J$-holomorphic if it satisfies the relation $d u+J(u) d u 1=0$. Since this should not produce any ambiguity, we will speak about "holomorphic" disks in both the integrable and the non integrable case, forgetting about the "pseudo" or $J$ prefix. The precise notions of contact and type we will work with are the following :

Definition 1. Let $u:(\Delta, 0) \rightarrow\left(\mathbb{R}^{2 n}, 0\right)$ be a (pseudo-)holomorphic disk mapping 0 to 0 , and regular i.e. such that $d u(0) \neq 0$. Its contact order $\delta_{0}(M, u)$ with $M$ at 0 is the degree of the first term in the Taylor expansion of $\phi \circ u$.

Definition 2. Let $\mathcal{D}_{\text {reg }}$ be the set of all germs of (pseudo-)holomorphic disks $u$ such that $u(0)=0$ and $d u(0) \neq 0$. The (regular) type of $M$ at 0 is the number

$$
\Delta_{\text {reg }}^{1}(M, 0)=\sup _{u \in \mathcal{D}_{\text {reg }}}\left(\delta_{0}(M, u)\right)
$$

Our aim is to compute the type of $M$ at 0 in terms of tangent vector fields on $M$. We propose two results in this direction, inspired from two classical results in complex analysis.

Our first result in this direction is a generalization of the classical results of 8 and 2 and computes the type in term of Lie brackets of "one" tangent vector field.

Theorem 1. Let $\delta=\mathbb{C} X$ be J-line in $T_{0} M$. The three following conditions are equivalent :

(1) There exists a smooth (J-)holomorphic disk $C=u(\Delta)$ tangent to $M$ at 0 with order $k+2$, with direction $\delta$ (i.e. $T_{0} C=\delta$ ).

(2) There exists a complex line sub-bundle $L$ of $T M$ such that $L_{0}=\delta$ and all the Lie brackets of length at most $k+1$ of sections of $L$ steel belong to $\delta$ at 0 .

(3) there exists a J-tangent vector field $X_{0}$ with $X_{0}(0) \in \delta$ such that all the Lie brackets of $X_{0}$ and $J X_{0}$ of length at most $k+1$ vanish at 0.

Moreover, if $\nabla$ is a symmetric connexion, $u$ and $X_{0}$ can be chosen such that at 0 : $X_{0}=\frac{\partial u}{\partial x}, \nabla_{X_{0}} X_{0}=\frac{\partial^{2} u}{\partial x^{2}}, \ldots, \nabla_{X_{0}}\left(\nabla_{X_{0}}\left(\ldots \nabla_{X_{0}} X_{0}\right)\right)=\frac{\partial^{k+1} u}{\partial x^{k+1}}$. 
Remark 1. If $L$ is involutive, i.e. (every where !) stable by Lie brackets, then by the Frobenius theorem, $M$ is foliated by $J$-holomorphic curves.

Our second result in the same direction uses "higher order" Levi forms, which are some kind of derivatives of the standard Levi form ; in contrast to the standard Levi form, they depend on the choice of a symmetric connexion.

Let us first discuss the Levi form itself. It is often defined on the complexification of $T M$, but we choose not to work in this setting to reduce the number of (almost) complex structures involved.

Thus we will use the following definition :

Definition 3. Let $T^{J} M=T M \cap J(T M)$ be the $J$-invariant part of $T M$. The function $L_{\phi}: T^{J} M \rightarrow \mathbb{R}$ defined by

$$
L_{\phi}(X)=d \phi(J[X, J X])
$$

is in fact a quadratic form on $T M$, called the Levi form associated to $\phi$. It depends on $\phi$ only up to multiplication by a non negative function.

The fact that $L(X)$ does not depend on the derivatives of $X$ is a straight forward computation. Let us just mention by the way the polar form associated to $L_{\phi}$ :

$$
\Theta_{\phi}(X, Y)=\frac{1}{2}(d \phi(J[X, J Y]+J[Y, J X])+1 d \phi(J[X, Y]+J[J X, J Y]))
$$

In particular, if $\alpha$ and $\beta$ are two real functions on $M$, then

$$
L_{\phi}((\alpha+\beta J) X)=\left(\alpha^{2}+\beta^{2}\right) L_{\phi}(X) .
$$

The classical relation between the Levi form and the second derivative of $\phi$ has to be modified in the non integrable case to take derivatives of the almost complex structure $J$ into account :

Proposition 4. Let $D_{\nabla}^{2} \phi$ denote the second derivative of $\phi$ with respect to $\nabla$. Then

$$
\left.L_{\phi}(X)=D_{\nabla}^{2} \phi(X, X)+D_{\nabla}^{2} \phi(J X, J X)+d \phi\left(\left(\nabla_{J X} J\right) X-\nabla_{X} J\right) J X\right)
$$

Remark 2. We will use a multiplicative notation for the derivatives, i.e. when differentiating many times in different directions, $\nabla_{X_{1}}\left(\nabla_{X_{2}}\left(\ldots \nabla_{X_{k-1}} X_{k}\right)\right)$ will be replaced by $X_{1} \cdot X_{2} \cdots X_{k}$, and if all the $X_{i}$ are the same, simply by $X^{k}$. We stress that this notation is somewhat misleading since this "product" is not associative.

The particular cases when the Levi form is non negative or positive are specially interesting.

Definition 5. The hyper-surface $M$ is said to be

- pseudoconvex if $L_{\phi} \geq 0$.

- strictly pseudoconvex if $L_{\phi}>0$.

The Levi form is a good tool to study the holomorphic disks tangent to $M$ at the first order, i.e. to check whether the type is 2 or grater than 2 :

Proposition 6. Consider the Levi form at the origin.

- If $\exists X \in T_{0}^{J} M, L_{\phi}(X)>0$, then there exits a smooth holomorphic disk $u: \Delta \rightarrow \mathbb{R}^{2 n}$ tangent to $M$ at 0 from the outside (i.e. lying in $M_{+}$).

- $\exists X \in T_{0}^{J} M, L_{\phi}(X)=0$ if and only if there exits a smooth holomorphic disk $u: \Delta \rightarrow \mathbb{R}^{2 n}$ tangent to $M$ at 0 with contact order $>2$.

Moreover, in each case, $X$ and $u$ can be chosen such that at $0: X=\frac{\partial u}{\partial x}$. 
Remark 3. This is just a computation, which will be detailed later on, as an introduction to the definition of higher order Levi forms. The existence of such holomorphic disk is useful to study properties of hyperbolicity for strictly pseudoconvex hyper-surfaces (see [7]).

The main result is as follows :

Theorem 2. Out of the derivatives of the Levi form (see definition 14 in section 4.1 for precise statement), one can compute functions $L^{p, q}:\left(\mathbb{R}^{2 n}\right)^{p+q+1} \rightarrow \mathbb{R}$ for all $p, q \in \mathbb{N}$ such that the two following conditions are equivalent :

(1) There exists a regular ( $J$-)holomorphic disk tangent to $M$ at 0 with order $k+2$

(2) There exists a complex tangent vector field $X \in \Gamma\left(T^{J} M\right)$ such that

$$
\forall(p, q), p+q \leq k-1, L^{p, q}\left(X(0), X^{2}(0), \ldots, X^{p+q+1}(0)\right)=0 .
$$

Moreover, $u$ and $X$ can be chosen such taht at $0: X=\frac{\partial u}{\partial x}, \ldots, X^{k+1}=\frac{\partial^{k} u}{\partial x^{k+1}}$; (recall that $X^{p}$ stands for $\underbrace{\nabla_{X}\left(\cdots\left(\nabla_{X}(X)\right)\right)}_{p})$.

Remark 4. This result generalizes the theorem of Bloom in $\mathbb{C}^{3}$ (see [1]) for pseudoconvex hyper-surface; but compare carefuly the definition of iterated derivatives of the levi form in our theorem and in 1].

The two theorems (1) and 2) rest on the comparison of jets of holomorphic disks and the appropriate notion of jets of vector fields on $M$.

\section{JETS OF DISKS AND TANGENT VECTOR FIELDS}

Definition 7. Define the $(p, q)$-th derivative of some vector field $X$ in $\mathbb{R}^{2 n}$, to be the vector $X$ differentiated $p$ times in its own direction and then $q$ times in the $J X$ direction :

$$
D_{X}^{p, q} X(0)=(J X)^{q} X^{p} \cdot X(0)=\underbrace{(J X \cdots(J X}_{q} \cdot \underbrace{(X \cdots(X}_{p} \cdot X)))(0)
$$

Definition 8. The collection of all the $(p, q)$-th derivatives of a vector field $X$ on $\mathbb{R}^{2 n}$ at 0 for $0 \leq p+q \leq k$ will be called its oder $k$ jet and denoted by $j_{0}^{k} X$. This defines a map

$$
\begin{aligned}
j_{0}^{k}: \Gamma\left(T \mathbb{R}^{2 n}\right) & \rightarrow \mathcal{J}_{0}^{k}\left(\mathbb{R}^{2 n}\right)=\left(\mathbb{R}^{2 n}\right)^{\frac{(k+1)(k+2)}{2}} \\
X & \mapsto\left(X(0), \ldots, D_{X}^{p, q} X(0), \ldots, D_{X}^{0, k} X(0)\right)
\end{aligned}
$$

and a jet $\xi \in \mathcal{J}_{0}^{k}\left(\mathbb{R}^{2 n}\right)$ is said to be realizable if it belongs to $\mathcal{J}_{0}^{k}(M)=j_{0}^{k}\left(\Gamma\left(T^{J} M\right)\right)$.

Given an holomorphic disk $u$ tangent to $M$, one would like to know whether or not it's jet at 0 can be realized as the jet of some vector field $X \in \Gamma\left(T^{J} M\right)$. This is what this section is devoted to.

Let us start with a basic observation :

Proposition 9. Let $X$ and $Y$ be two J-tangent vector fields such that $j_{0}^{k}(X)=$ $j_{0}^{k}(Y)$. Then for all $p, q \in \mathbb{N}$ with $p+q=k+1$, we have

$$
D_{X}^{p, q} X(0)-D_{Y}^{p, q} Y(0) \in T^{J} M
$$

Proof. One has to prove the two equalities

$$
\begin{aligned}
d \phi\left(D_{X}^{p, q} X(0)-D_{Y}^{p, q} Y(0)\right) & =0 \\
d \phi\left(J\left[D_{X}^{p, q} X(0)-D_{Y}^{p, q} Y(0)\right]\right) & =0 .
\end{aligned}
$$


Let us compute $D_{X}^{p, q}(d \phi(X))$. On one hand, it is 0 (since $X \in T M$ ). On the other hand, the development of $D_{X}^{p, q}(d \phi(X))$ is a sum of terms of the form

$$
D^{\alpha} \phi\left(D_{X}^{p_{1}, q_{1}} X(0), \ldots, D_{X}^{p_{\alpha}, q_{\alpha}} X(0)\right)
$$

If $p_{i}+q_{i} \leq k$, then this term is the same as

$$
D^{\alpha} \phi\left(D_{Y}^{p_{1}, q_{1}} Y(0), \ldots, D_{Y}^{p_{\alpha}, q_{\alpha}} Y(0)\right)
$$

Thus $0-0=D_{X}^{p, q}(d \phi(X))-D_{Y}^{p, q}(d \phi(Y))=d \phi\left(D_{X}^{p, q} X-D_{Y}^{p, q} Y\right)$.

The equality (6) is obtained along the same lines (eventually taking the derivatives of $J$ into account) using $D_{X}^{p, q}(d \phi(J X))=0$ since $J X \in T M$.

Realizability of jets can then be recursively tested as follows :

Proposition 10. Let $\xi \in \mathcal{J}_{0}^{k+1}\left(\mathbb{R}^{2 n}\right)$. Let $[\xi]_{k}$ be its $\mathcal{J}_{0}^{k}\left(\mathbb{R}^{2 n}\right)$-component, and $\left(\xi_{k+1,0}, \ldots, \xi_{p, q}, \ldots, \xi_{0, k+1}\right)$ its homogeneous part of degree $k+1$. Then

$$
\exists X \in \Gamma\left(T^{J} M\right), j_{0}^{k+1}(X)=\xi
$$

if and only if

$$
\left\{\begin{array}{l}
\exists X_{1} \in \Gamma\left(T^{J} M\right), j_{0}^{k}\left(X_{1}\right)=[\xi]_{k} \\
\forall(p, q), p+q=k+1, \quad \xi_{p, q}-\left[j_{0}^{k+1}\left(X_{1}\right)\right]_{p, q} \in T_{0}^{J} M
\end{array}\right.
$$

In other words, if you take a realizable $k+1$-jet, then the $N$ and $J N$ components of its maximal degree part are completely determined by its $k$-order part, but the $T^{J} M$ component can be anything.

Proof. (7) $\Rightarrow(8)$ is obvious. So let us start with $X_{1}$ and the compatibility condition (8). Let $\left(T_{1}, \ldots, T_{n-1}\right)$ be a (complex) basis of $T^{J} M$ and let $X=X_{1}+\sum \lambda_{i} T_{i}$, where the $\lambda_{i}$ are complex valued functions on $M$, all the derivatives of which vanish at 0 up to order $k$, and such that $\lambda_{i}(0)=1$. Then if $p+q \leq k, D_{X}^{p, q} X=$ $D_{X_{1}+\sum \lambda_{i} T_{i}}\left(X+\sum \lambda_{i} T_{i}\right)$ is the sum of $D_{X_{1}^{p}}^{p, q} X_{1}$ and of terms involving derivatives of the $\lambda_{i}$ of order at most $k$. Therefore at 0 we have

$$
\forall(p, q), p+q \leq k, \quad D_{X}^{p, q} X(0)=D_{X_{1}}^{p, q} X_{1}(0)
$$

For $p+q=k+1$, the $k+1$-th derivatives of $\lambda_{i}$ appear in the following terms :

$$
\begin{aligned}
D_{X}^{p, q} X(0) & =D_{X_{1}}^{p, q} X_{1}(0)+\sum\left(D_{X_{1}}^{p, q} \lambda_{i}\right)(0) T_{i}(0) \\
& =D_{X_{1}}^{p, q} X_{1}(0)+\sum D_{0}^{k+1} \lambda_{i}\left(J X_{1}, \ldots, J X_{1}, X_{1}, \ldots, X_{1}\right) T_{i}(0)
\end{aligned}
$$

which proves that the $k+1$ degree part of $j_{0}^{k}\left(X_{1}\right)$ can only be modified in the $T^{J} M$ direction, and all the modification in this direction are possible since any $\left(\alpha_{k+1,0}, \alpha_{k, 1}, \ldots, \alpha_{0, k+1}\right) \in \mathbb{C}^{k+2}$ can be realized as the derivatives of a complex valued function in the directions $X_{1}$ and $J X_{1}$ (provided that $X_{1} \neq 0$ ) : $\forall\left(\alpha_{k+1,0}, \alpha_{k, 1}, \ldots, \alpha_{0, k+1}\right) \in \mathbb{C}^{k+2} \quad \exists \lambda: M \rightarrow \mathbb{C}$ such that

$$
\left\{\begin{array}{l}
\lambda(0)=1 \\
\forall r \leq k, \quad D_{0}^{r} \lambda=0 \\
\forall(p, q), p+q=k+1, \quad D_{0}^{k+1} \lambda\left(J X_{1} \ldots J X_{1}, X_{1} \ldots X_{1}\right)=\alpha_{p, q}
\end{array}\right.
$$

Corollary 11. There exists a regular holomorphic disk $u: \Delta \rightarrow \mathbb{R}^{2 n}$ tangent to $M$ at 0 with order $k+2$ (i.e. $\phi \circ u(z)=O\left(z^{k+2}\right)$ ) if and only if the $k+1$-jet of $u$ at 0 is realizable, i.e.

$$
\exists X \in \Gamma\left(T^{J} M\right), \quad \forall(p, q), p+q \leq k, \quad D_{X}^{p, q} X(0)=\frac{\partial^{p+q} u}{\partial x^{p} \partial y^{q}} \frac{\partial u}{\partial x}(0) .
$$


Proof. Let $u$ be a disk tangent to $M$ with order $k+3, \xi$ the $k$-jet of $\frac{\partial u}{\partial x}$ at 0 (i.e. $\left.\xi_{p, q}=\frac{\partial^{p+q+1} u}{\partial x^{p+1} \partial y^{q}}(0)\right)$. By induction, there exists a $J$ tangent vector field $X_{1}$ realizing the $k$-jet of $\frac{\partial u}{\partial x}$. Then, comparing the developments of

$$
\frac{\partial^{k+1}}{\partial x^{p} \partial y^{q}}\left[d \phi\left(\frac{\partial u}{\partial x}\right)\right](0) \quad \text { and } \quad D_{X_{1}}^{p, q}\left[d \phi\left(X_{1}\right)\right](0)
$$

for $p+q=k+1$, it turns out that all the terms are the same except maybe the terms where no derivative is applied to $d \phi$. Thus, we have :

$$
\frac{\partial^{k+1}}{\partial x^{p} \partial y^{q}}\left[d \phi\left(\frac{\partial u}{\partial x}\right)(0)\right](0)-D_{X_{1}}^{p, q}\left[d \phi\left(X_{1}\right)\right](0)=d \phi\left[\frac{\partial^{k+1}}{\partial x^{p} \partial y^{q}} \frac{\partial u}{\partial x}(0)-D_{X_{1}}^{p, q} X_{1}(0)\right] .
$$

On the other hand, $\frac{\partial^{k+1}}{\partial x^{p} \partial y^{q}}\left[d \phi\left(\frac{\partial u}{\partial x}\right)\right](0)=0$ since $\phi \circ u(z)=O\left(z^{k+3}\right)$, and $D_{X_{1}}^{p, q}\left[d \phi\left(X_{1}\right)\right](0)=$ 0 since $X_{1} \in T M$. Thus we have

$$
d \phi\left(\xi_{p, q}-\left[j_{0}^{k+1} X_{1}\right]_{p, q}\right)=0 .
$$

Comparing in the same way

$$
\frac{\partial^{k+1}}{\partial x^{p} \partial y^{q}}\left[d \phi\left(J(u) \frac{\partial u}{\partial x}\right)\right](0) \quad \text { and } \quad D_{X_{1}}^{p, q}\left[d \phi\left(J X_{1}\right)\right](0)
$$

we get

$$
\frac{\partial^{k+1}}{\partial x^{p} \partial y^{q}}\left[d \phi\left(\frac{\partial u}{\partial y}\right)(0)\right](0)-D_{X_{1}}^{p, q}\left[d \phi\left(J X_{1}\right)\right](0)=d \phi\left[J \frac{\partial^{k+1}}{\partial x^{p} \partial y^{q}} \frac{\partial u}{\partial x}(0)-J D_{X_{1}}^{p, q} X_{1}(0)\right] .
$$

and finally

$$
d \phi\left(J \xi_{p, q}-J\left[j_{0}^{k+1} X_{1}\right]_{p, q}\right)=0 .
$$

The relations (12) and (14) and the proposition 10 now proves that $X_{1}$ can be modified into a new complex tangent vector field $X$ to have :

$$
j_{0}^{k+1} X=\xi \text {. }
$$

Start now with $u$ and $X \in T^{J} M$ such that $\forall(p, q), p+q \leq k, \frac{\partial^{p+q+1} u}{\partial x^{p+1} y^{q}}(0)=$ $D_{X}^{p, q} X(0)$ and compute $\frac{\partial^{p+q} \phi \circ u}{\partial x^{p} \partial y^{q}}(0)$ for $p+q \leq k+1$. The main tool is again the relations (11) and (13). If $p>1$ we have

$$
\begin{aligned}
\frac{\partial^{p+q}}{\partial x^{p} \partial y^{q}}[\phi \circ u](0) & =\frac{\partial^{p+q}}{\partial x^{p-1} \partial y^{q}}\left[d \phi\left(\frac{\partial u}{\partial x}\right)\right](0) \\
& =D_{X}^{p-1, q}[d \phi(X)](0)=0
\end{aligned}
$$

and if $p=0$ :

$$
\begin{aligned}
\frac{\partial^{q}}{\partial y^{q}}[\phi \circ u](0) & =\frac{\partial^{q-1}}{\partial y^{q-1}}\left[d \phi\left(J \frac{\partial u}{\partial x}\right)\right](0) \\
& =D_{X}^{0, q-1}[d \phi(J X)](0)=0
\end{aligned}
$$

Finally, we have $\phi \circ u(z)=O\left(z^{k+2}\right)$.

\section{Regular type and Lie algebra of Line Bundles}

\subsection{Commutativity of vector fields at one point.}

Definition 12. A complex tangent vector field $X$ is said to commute at 0 up to order $k$ if and only if $\forall m \leq k, \forall X_{1}, \ldots, X_{m} \in\{X, J X\}$ and $\forall \sigma \in \mathfrak{S}_{m}$ we have $X_{\sigma(1)} \cdot X_{\sigma(2)} \cdots X_{\sigma(k)}=X_{1} \cdot X_{2} \cdots X_{k}$.

Of course, we can use the group structure of $\mathfrak{S}_{k}$ and the properties of the Lie brackets to reduce attention only to a few permutations : 
Proposition 13. Let $X$ be a complex tangent vector fields. The four following conditions are equivalent :

(1) $X$ commutes up to order $k$ at 0 .

(2) $\forall p, q / p+q \leq k$,

$$
\underbrace{J X \cdots J X}_{q} \cdot \underbrace{X \cdots X}_{p} \cdot J X=\underbrace{J X \cdots J X}_{q+1} \cdot \underbrace{X \cdots X}_{p}
$$

(3) All the Lie brackets of $X$ and $J X$ up to length $k$ vanish at 0.

(4) $\forall m \leq k-2, \forall X_{1} \ldots X_{m} \in\{X, J X\}, X_{1} \cdots X_{m} \cdot[X, J X]=0$

Proof. (11) $\Rightarrow$ (2) is clear.

(2) $\Rightarrow$ (11) rests on the remark that if $X$ commutes up to order $k-1$, then the relations of (11) for $m=k$ and $\sigma(k)=k$ are automatic :

$$
X_{1} \cdots X_{k-1} \cdot X_{k}=\sum_{\substack{1 \leq \alpha \leq k-1 \\ I_{1} \cup \cdots \cup I_{\alpha}=\{1 \ldots n\} \\ I_{i} \cap I_{j}=\emptyset}} D^{\alpha} X_{k}\left(X^{I_{1}}, \ldots, X^{I_{\alpha}}\right)
$$

where $X^{I_{i}}=X_{i_{1}} \cdots X_{i_{r}}$ when $I_{i}=\left\{i_{1}<\cdots<i_{r}\right\}$.

The group $\mathfrak{S}_{k-1}$ acts transitively on the indexing set, and since $X$ commutes up to order $k-1, X^{I_{i}}$ does not depend on the order used on $I_{i}$. We conclude that $X_{1} \cdots X_{k}=X_{\sigma(1)} \cdots X_{\sigma(k-1)} \cdot X_{k}$ for all $\sigma \in \mathfrak{S}_{k-1}$. This ends the proof since the permutations corresponding to the relation (2) and $\mathfrak{S}_{k-1}$ generate $\mathfrak{S}_{k}$.

(11) $\Rightarrow$ (3) is clear.

(3) $\Rightarrow$ (4) : suppose that all the Lie brackets of $X$ and $J X$ vanish at 0 up to length $k$, and, by induction, that all the derivatives of $[X, J X]$ in the $X$ and $J X$ directions vanish at 0 up to order $k-3$. Then in the development of $\left[X_{1}\left[\ldots X_{k-2}[X, J X]\right]\right]$, all the terms vanish but the term where $[X, J X]$ is differentiated $k-2$ times:

$$
0=\left[X_{1}\left[\ldots X_{k-2}[X, J X]\right]\right]=X_{1} \cdots X_{k-2} \cdot[X, J X]
$$

(41) $\Rightarrow$ (11) : it is enough to prove the relation when $\sigma$ is a transposition $(i, i+1)$. But $X_{1} \cdots X \cdot(J X) \cdots X_{m}-X_{1} \cdots(J X) \cdot X \cdots X_{m}=X_{1} \cdots[X, J X] \cdots X_{m}$, and all the terms in the development of this last expression involve a derivative of order at most $m-2$ of $[X, J X]$ in the $X$ and $J X$ directions : thus it is null.

We can now come to the proof of theorem 1

\subsection{Proof of theorem 1}

Proof of (11) $\Rightarrow(3)$ in theorem 1 : Start with a regular disk $u:(\Delta, 0) \rightarrow\left(\mathbb{R}^{2 n}, 0\right)$ tangent to $M$ with order $k+2$. Then, by corollary 11$]$ there exists a complex tangent vector field $X$ such that $\forall(p, q), p+q \leq k, \frac{\partial^{p+q}}{\partial x^{p} \partial y^{q}} \frac{\partial u}{\partial x}(0)=D_{X}^{p, q} X(0)$. Let us check that this $X$ commutes up to order $k+1$ at 0 , i.e. that $D_{X}^{p, q}(J X)(0)=D_{X}^{p-1, q+1} X(0)$ for $p+q \leq k$.

We have the relation

$$
D_{X}^{p, q}(J X)=\sum_{\substack{a+c=p \\ b+d=q}} C_{p}^{a} C_{q}^{b}\left(D_{X}^{a, b} J\right) D_{X}^{c, d} X
$$

Differentiating $\frac{\partial u}{\partial y}=J(u) \frac{\partial u}{\partial x}$ with respect to $x p$ times and to $y q$ times, we obtain

$$
\frac{\partial^{p+q}}{\partial x^{p} \partial y^{q}} \frac{\partial u}{\partial y}=\sum_{\substack{a+c=p \\ b+d=q}} C_{p}^{a} C_{q}^{b}\left(\frac{\partial^{a+b}}{\partial x^{a} \partial y^{b}}(J(u))\right) \frac{\partial^{c+d}}{\partial x^{c} \partial y^{d}} \frac{\partial u}{\partial x}
$$


fom which we deduce that :

$$
\begin{aligned}
D_{X}^{p, q}(J X)(0) & =\frac{\partial^{p+q}}{\partial x^{p} \partial y^{q}} \frac{\partial u}{\partial y}(0) \\
& =\frac{\partial^{p+q}}{\partial x^{p-1} \partial y^{q+1}} \frac{\partial u}{\partial x}(0) \\
& =D_{X}^{p-1, q+1} X(0)
\end{aligned}
$$

This ends the proof of (1) $\Rightarrow(3)$ in theorem 1

Proof of $(3) \Rightarrow$ (1) in theorem 1): Let now $X$ be a vector field commuting up to order $k+1$ at 0 . By an argument of J.-C. Sikorav [10, there exists a germ $u: \Delta \rightarrow \mathbb{R}^{2 n}$ of $J$-holomorphic disk such that $\forall m \leq k+1, \frac{\partial^{m} u}{\partial x^{m}}(0)=X^{m}(0)$. Since $X$ commutes, one can easily check that $\frac{\partial^{p+q}}{\partial x^{p} \partial y^{q}} \frac{\partial u}{\partial x}=D_{X}^{p, q} X(0)$ and then apply proposition 11. Proof of (2) $\Leftrightarrow(3)$ in theorem [1]: The basic remark here is that if $X$ is replaced by $(\alpha+\beta J) X$ where $\alpha$ and $\beta$ are real valued functions on $M$, then an iterated Lie bracket of $X$ and $J X$ of length $k$ is affected by addition of a linear combination of strictly shorter Lie brackets. Therefore, if $X$ commutes up to roder $k+1$ at 0 , then the line subbundle generated by $X$ is stable at 0 up to order $k+1$. In the other direction, if a line bundle is stable at 0 up to order $k+1$, then choosing a local non vanishing section $X$ of it, and suitable functions $\alpha$ and $\beta$, we obtain a vector field commuting at 0 up to order $k+1$.

\section{HighER ORDER LEVI FORMS}

4.1. Definition : As an introduction, let us discuss the proof of proposition 6 The main tool here is the relationship between the Levi form and laplacian of $\phi \circ u$ for tangent disks $u$.

Suppose that $X \in T_{0}^{J} M$ satisfies $L_{\phi}(X)>0$. Recall that from an argument of Sikorav [10, all the derivatives $\frac{\partial^{k} u}{\partial x^{k}}(0)$ of a holomorphic disk can be chosen arbitrarily, i.e. for all $X_{1}, \ldots, X_{k} \in \mathbb{R}^{2 n}$ there exists a germ of holomorphic disk such that $X_{i}=\frac{\partial^{i} u}{\partial x^{i}}(0)$.

Let $u: \Delta \rightarrow \mathbb{R}^{2 n}$ be a (germ of) holomorphic disk such that $u(0)=0, \frac{\partial u}{\partial x}(0)=$ $X$. In the Taylor expansion of $\phi \circ u$, use the fact that $u$ is holomorphic, that is $\frac{\partial u}{\partial y}=J(u) \frac{\partial u}{\partial x}$, to express all the deirvatives of $u$, as derivatives with respect to $x$ only. We get :

$$
\phi \circ u(z)=a_{2,0} x^{2}+a_{1,1} x y+a_{0,2} y^{2}+o\left(x^{2}+y^{2}\right)
$$

where

$$
\begin{aligned}
& a_{2,0}=\underbrace{D^{2} \phi(X, X)}_{a}+D \phi\left(\frac{\partial^{2} u}{\partial x^{2}}\right) \\
& a_{1,1}=\underbrace{D^{2} \phi(J X, X)+D \phi((X \cdot J) X)}_{b}+D \phi\left(J \frac{\partial^{2} u}{\partial x^{2}}\right) \\
& a_{0,2}=\underbrace{D^{2} \phi(J X, J X)+D \phi(((J X) \cdot J) X-(X \cdot J) J X)}_{c}-D \phi\left(\frac{\partial^{2} u}{\partial x^{2}}\right)
\end{aligned}
$$

Recall from (4) that $L_{\phi}(X)=D^{2} \phi(X, X)+D^{2} \phi(J X, J X)+D \phi(((J X) \cdot J) X-$ $(X \cdot J) J X)$ :

$$
L_{\phi}(X)=a_{2,0}+a_{0,2}=\Delta(\phi \circ u)(0) .
$$

Since $\frac{\partial^{2} u}{\partial x^{2}}(0)$ can be chosen arbitrarily, we can in particular choose $d \phi\left(\frac{\partial^{2} u}{\partial x^{2}}\right)(0)$ (i.e. its $N$-component) and $d \phi\left(J \frac{\partial^{2} u}{\partial x^{2}}\right)(0)$ (i.e. its $J N$ component) independently. 
The choices

$$
d \phi\left(\frac{\partial^{2} u}{\partial x^{2}}\right)(0)=\frac{c-a}{2} \quad d \phi\left(J \frac{\partial^{2} u}{\partial x^{2}}\right)(0)=-b
$$

lead to the formula

$$
\phi \circ u(z)=\frac{L_{\phi}(X)}{2}\left(x^{2}+y^{2}\right)+o\left(|z|^{2}\right)
$$

If $L_{\phi}(X)>0, u$ is clearly tangent to $M$ from the outside.

If $L_{\phi}(X)=0$, then the contact order of $u$ with $M$ is at least 3 .

If $u$ has a contact of order at least 3 with $M$, then $a_{2,0}=a_{1,1}=a_{0,2}=0$ and the relation (17) proves that taking $X=\frac{\partial u}{\partial x}(0) \in T_{0}^{J} M$, we have $L_{\phi}(X)=0$.

This ends the proof of theorem [

The following definition of the higher order Levi forms derive from very similar ideas in the study of the higher order Taylor expansion of $\phi \circ u$ : the pairwise sums of the coefficients are related to the derivatives of $\Delta(\phi \circ u)$, and thus to derivatives of the Levi form.

Let $u$ be a germ of holomorphic disk at 0 . Using the relation $\frac{\partial u}{\partial y}=J(u) \frac{\partial u}{\partial x}$ and eventually derivatives of it, it is possible to express $\frac{\partial^{k} \Delta(\phi \circ u)}{\partial x^{p} \partial y^{q}}$ by means of derivatives of $u$ in the $x$ direction only, and this process is purely algebraic :

Definition 14. For all $(p, q)$, there exists a polynomial function $L^{p, q}:\left(\mathbb{R}^{2 n}\right)^{p+q+1} \rightarrow$ $\mathbb{R}$, called the $(p, q)$-th Levi form which depends on (the derivatives of) $\phi$ and $J$ only, such that for all germ of holomorphic disk $u$ at 0 :

$$
\frac{\partial^{p+q}}{\partial x^{p} \partial y^{q}} \Delta(\phi \circ u)=L^{p, q}\left(\frac{\partial u}{\partial x}, \ldots, \frac{\partial^{p+q+1} u}{\partial x^{p+q+1}}\right)
$$

At first sight, it seems that $L^{p, q}$ should depend on the derivatives of $u$ up to order $p+q+2$ and not only $p+q+1$ as mentioned in this definition. In fact, the derivatives of $u$ of order $p+q+2$ involoved in the computation of $\frac{\partial^{p+q}}{\partial x^{p} \partial y^{q}} \Delta(\phi \circ u)$ apear only in the following term : $d \phi\left(\frac{\partial^{p+q+2} u}{\partial x^{p+2} \partial y^{q}}+\frac{\partial^{p+q+2} u}{\partial x^{p} \partial y^{q+2}}\right)$ which is null in the integrable case, and involves only derivatives of $u$ of order at most $p+q+1$ in the non integrable one.

Especially in the almost complex case, the explicit computation of $L^{p, q}$ is of course a bit tedious, but we stress that it is not more complicated than the computation of the derivatives of some compound function. More precisely, in the integrable case, $L^{p, q}$ can be obtained by the following "recipe" : formally compute the $(p, q)$-th derivative of $\Delta(\phi \circ u)$, and replace the $(\alpha, \beta)$-derivatives of $u$ by $J^{\beta} X_{\alpha+\beta}$.

Another way to think about $L^{p, q}\left(X, X^{2}, \ldots, X^{k}\right)$ for a given vector field $X$, is to formally differentiate the usual Levi form, $p$ times in the $X$ direction, then $q$ times in the $J X$ direction, and then "force" the commutation of $X$ and $J X$ by replacing all $(J X)^{\beta} X^{\alpha}$ by $J^{\beta} X^{\alpha+\beta}$. In the non integrable case, this commutation relation involves of course some derivatives of $J$.

For instance, in the integrable case :

$$
\begin{aligned}
L^{0,0}\left(X_{1}\right)=D^{2} \phi\left(X_{1}, X_{1}\right)+D^{2} \phi\left({ }_{1} X_{1},{ }_{1} X_{1}\right) \\
L^{1,0}\left(X_{1}, X_{2}\right)=D^{3} \phi\left(X_{1}, X_{1}, X_{1}\right)+D^{3} \phi\left(X_{1},{ }_{1} X_{1},{ }_{1} X_{1}\right)+ \\
+2 D^{2} \phi\left(X_{2}, X_{1}\right)+2 D^{2} \phi\left({ }_{1} X_{2},{ }_{1} X_{2}\right) \\
L^{0,1}\left(X_{1}, X_{2}\right)=D^{3} \phi\left({ }_{1} X_{1}, X_{1}, X_{1}\right)+D^{3} \phi\left({ }_{1} X_{1},{ }_{1} X_{1},{ }_{1} X_{1}\right)+ \\
+ \\
+2 D^{2} \phi\left(1 X_{2}, X_{1}\right)-2 D^{2} \phi\left(X_{2},{ }_{1} X_{2}\right)
\end{aligned}
$$


By construction, if $u$ is a holomorphic disk, the extended Levi forms above are related to the Taylor expansion of $\phi \circ u$ at 0 in the following way : write the homogeneous part of degree $k$ as

$$
[\phi \circ u(z)]_{k}=a_{k, 0} x^{k}+a_{k-1,1} x^{k-1} y+\cdots+a_{0, k} y^{k} .
$$

Then

$$
a_{i+2, j}+a_{i, j+2}=L^{i, j}\left(\frac{\partial u}{\partial x}, \ldots, \frac{\partial^{k-1} u}{\partial x^{k-1}}\right)
$$

We can now turn to the proof of theorem 2

\subsection{Proof of theorem 2 ,}

Proof of $(1) \Rightarrow(2)$ in theorem 2 : Start with a holomorphic disk $u$ tangent to $M$ at 0 with order $k+2$. Then the relation (20) proves that for all $(i, j), i+j+2 \leq k+1$ we have

$$
L^{i, j}\left(\frac{\partial u}{\partial x}, \ldots, \frac{\partial^{i+j+1} u}{\partial x^{i+j+1}}\right)=0 .
$$

On the other hand, the corollary 11 provides us with a $J$-tangent vector field $X$ such that $\forall m \leq k+1, \frac{\partial^{m} u}{\partial x^{m}}(0)=X^{m}(0)$, and therefore, $\forall(i, j), i+j \leq k-1$ :

$$
L^{i, j}\left(X(0), \ldots, X^{i+j+1}(0)\right)=0 .
$$

Proof of (2) $\Rightarrow$ (11) in theorem 2]: Start with a $J$-tangent vector field $X$ such that $\forall(i, j), i+j \leq k-1, L^{i, j}\left(X(0), \ldots, X^{i+j+1}(0)\right)=0$. Choose a germ $u$ of holomorphic disk at 0 such that

$$
\forall m \leq k+1, \frac{\partial^{m} u}{\partial x^{m}}(0)=X^{m}(0)
$$

Suppose by induction that $u$ is tangent to $M$ with order $k+1$. The Taylor expansion of $\phi \circ u$ has the form

$$
\phi \circ u(z)=a_{k+1,0} x^{k+1}+a_{k, 1} x^{k} y+\cdots+a_{0, k+1} y^{k+1}+o\left(|z|^{k+1}\right) .
$$

and because of relation (20), we have

$$
\forall(i, j), i+j+2=k+1, a_{i+2, j}=-a_{i, j+2} .
$$

Using (23), one can easily compute $a_{k+1,0}$ :

$$
\begin{aligned}
a_{k+1,0} & =\frac{\partial}{\partial x^{k}} d \phi\left(\frac{\partial u}{\partial x}\right)(0) \\
& =X^{k}(d \phi(X))(0)=0 .
\end{aligned}
$$

To compute $a_{k, 1}$, let us first remark that the computation of $X^{k} \cdot(J X)(0)$ is a sum of terms involving a derivative of $J$ in the $\left(X^{\alpha}\right)_{\alpha<k+1}$ directions and one of the $\left(X^{\alpha}\right)_{\alpha \leq k+1}$. The computation of $\frac{\partial}{\partial x^{k}} d \phi\left(J(u) \frac{\partial u}{\partial x}\right)(0)$ leads to the same sum where the $X^{\alpha}$ are replaced by the $\frac{\partial^{\alpha} u}{\partial x^{\alpha}}$, and thus :

$$
\begin{aligned}
a_{k, 1} & =\frac{\partial}{\partial x^{k}} d \phi\left(J(u) \frac{\partial u}{\partial x}\right)(0) \\
& =X^{k}(d \phi(J X))(0)=0
\end{aligned}
$$

The relations (24), (25) and (26) prove that $\phi \circ u=O\left(z^{k+2}\right)$, ending the proof of the theorem. 


\section{BASE POINT DEPENDENCY IN LOWER DIMENSIONS}

In general, even in the pseudo-convex case, the type is not an upper continuous function on $M$ : it is not even locally bounded (see [6] for striking examples). However, in complex dimension 2, it is known to be upper-continuous in the integrable case. In the first part of this section, we give a proof of this fact with the point of vue developped all along this paper ; in particular, the proof still apply to the almost complex case.

In the second part of this section, we discuss a particular case in complex dimension 3 : if the Levi form has constant signature, the type is an upper-continuous function of the base point.

\subsection{Upper continuity in dimension 2 .}

Proposition 15. In complex dimension 2 (real dimension 4), the regular type of an hyper-surface $M$ is an upper-continuous function on $M$.

The basic idea of the proof is to translate $\Delta_{\text {reg }}^{1}(p) \geq k$ in terms of jets : the functions $L^{p, q}$ are defined on a space of jets, and since they are invariant under rescaling, we can reduce to a compact subset. The set $\left\{L^{p, q}=0, p+q \leq k\right\}$ is then compact and a sequence of "good" jets above a converging sequence $p_{n}$ in $M$ should therefore (sub-)converge to a "good" jet at $p_{\infty}$. The point is that the set of realizable jets is not closed, since if the first component of the jet vanishes, all the remaining components should vanish as well.

This phenomenon occurs when the sequence of curves catches a singularity in the limit. In terms of jets, if we rescale our jets so that the first component has always length 1 , this means that one component explodes in the limit. Fortunately, in dimension 2 (real dimension 4), there is no room for that, and the phenomenon can be controlled.

Proof. Let us first concentrate on one point where the type is $k+2$. Let $X$ be a germ of $J$-tangent vector field at this point on which all the Levi forms vanish up to order $k-1$.

Let $u$ be a germ of holomorphic disk at 0 such that $\forall m \leq k+1, \frac{\partial^{m} u}{\partial x^{m}}(0)=X^{m}(0)$. Then, $u$ has contact order $k+2$ with $M$ and thus, for any germ $\theta$ of holomorphic function with $\theta(0)=0, \theta^{\prime}(0) \neq 0$, the disk $v(z)=u \circ \theta(z)$ also has contact order $k+2$. Suitably choosing the jet of $\theta$, and computing the jet of $v$ at 0 , we obtain that in fact any $Y=(\alpha+\beta J) X$ where $\alpha$ and $\beta$ are real valued functions, i.e. any section of $T^{J} M$, satisfy the relations $L^{p, q}\left(Y, \ldots, Y^{p+q+1}\right)=0$ for $p+q \leq k-1$.

Let now $X$ be a local non vanishing section $X$ of $T^{J} M$ near 0 , and $p_{n}$ a sequence of points on $M$ converging to 0 . If each $p_{n}$ is of type at least $k+2$, then the functions $L^{p, q}\left(X, \ldots, X^{p+q+1}\right)$ vanish at each $p_{n}$, and thus at 0 . therefore

$$
\Delta_{\text {reg }}^{1}\left(\lim p_{n}\right) \geq \limsup \Delta_{\text {reg }}^{1}\left(p_{n}\right) \text {. }
$$

5.2. Case of dimension 3 with non degenerate Levi form. The behaviour of the jet can also be controlled in some cases if the Levi form is not degenerated :

Theorem 3. If $n=3$ and the Levi form on $M$ is (every where) non degenerate then $\Delta_{\mathrm{reg}}^{1}(p)$ is an upper continuous function of $p$.

Proof. If the Levi form is definite positive (or negative) then $\Delta_{\text {reg }}^{1}(p)=2$ every where.

If it is not, its signature is $(1,1)$, and the type is at least 3 every where. Let $(X, Y)$ be a field of bases of $T^{J} M$ in which $L=\left(\begin{array}{ll}0 & 1 \\ 1 & 0\end{array}\right)$. The coordinates are chosen such that $(X(0), Y(0), N(0))$ is the canonical basis of $\mathbb{C}^{3}$. Suppose that the type is 
at least $k+2$ at 0 : let $u$ be a regular disk with contact $k+2$ and $Z$ a complex vector field on $M$ realizing the jet of $\frac{\partial u}{\partial x}$ up to order $k$.

In the integrable case, we can reparametrise $u$, so that $u(z)=(z, h(z), g(z))$. In the non integrable case, this is not possible, but the first coordinate of $u$ can still be brought to the form $z+r(z)$ where $r(z)=o(z)$ is a function whose derivatives are controlled by the derivatives of $J$, and the derivatives of the other coordinates.

Let us decompose $Z^{k}(0)$ in the base $(X, J X, Y, J Y, N, J N): Z^{k}=\alpha X(0)+$ $\beta Y(0)+\gamma N(0)$, where $\alpha, \beta, \gamma$ are complex valued functions.

The $X$-component of $Z^{k}$ is controlled by the $C^{k}$ norm of $J$ and the other components of $Z^{k}$.

We already proved (see the proof of 10 ) that the $N$ and $J N$ components of $Z^{k}$ can be expressed as a combination of derivatives of $\phi$ (of length at most $k$ ) and all the $Z^{i}$ for $i<k$ : we conclude that

$$
|\gamma| \leq\|\phi\|_{C^{k}} \sup _{i<k}\left\|Z^{i}\right\|^{k / i}
$$

To control the $Y$ component of $Z^{k}$ at 0 , remark that in $L^{k-1,0}\left(Z, \ldots, Z^{k}\right)$, the only term involving $Z^{k}$ is $L\left(Z^{k}, Z\right)$ :

$$
L^{k-1,0}\left(Z, \ldots, Z^{k}\right)=2 L\left(Z^{k}, Z\right)+P_{\phi}\left(Z, \ldots, Z_{k-1}\right)
$$

where $P_{\phi}\left(Z, \ldots, Z_{k-1}\right)$ is a homogeneous polynomial in $Z, \ldots, Z_{k-1}$ of degree $k+1$, whose coefficients come from derivatives of $\phi$ and $J$ up to order $k$.

We obtain that $L\left(\beta_{1} Y+\beta_{2} J Y, X\right)=A_{\phi}\left(Z, \ldots, Z_{k-1}\right)$ where $A_{\phi}\left(Z, \ldots, Z_{k-1}\right)$ is an homogeneous polynomial in $\left(Z, \ldots, Z_{k-1}\right)$ controlled by the $C^{k}$ norm of $\phi$ and $J$.

In the same way, we can prove that $L\left(\beta_{1} Y+\beta_{2} J Y, J X\right)=B_{\phi}\left(Z, \ldots, Z_{k-1}\right)$ where $B_{\phi}$ is again controlled by the $C^{k}$ norms of $\phi$ and $J$.

Finally

$$
\begin{cases}\beta_{1} L(Y, X)+\beta_{2} L(J Y, X) & =A_{\phi}\left(Z, \ldots, Z_{k-1}\right) \\ \beta_{1} L(Y, J X)+\beta_{2} L(J Y, J X) & =B_{\phi}\left(Z, \ldots, Z_{k-1}\right)\end{cases}
$$

and since $L(J Y, X)=-L(J Y, X)$, the determinant of this system is at least $L(Y, X)^{2}=1$. As a consequence, $|\beta|$ is controlled by the $C^{k}$ norms of $\phi$ and $J$, and the norms of $\left(Z, \ldots, Z_{k-1}\right)$ : there exists a contant $M$, depending only on the $C^{k}$ norms of $\phi$ and $J$ such that

$$
\left\|Z^{k}\right\| \leq M \sup _{i<k}\left\|Z^{i}\right\|^{k / i}
$$

By induction, we obtain a collection of constants $M_{i}$ such that at 0 :

$$
\forall i \leq k,\left\|Z^{i}\right\| \leq M_{i}\|Z\|^{i} .
$$

This inequality implies that if we consider a converging sequence $p_{n}$ of points of type at least $k+2$ in $M$, we can find a sequence of realizable jets $\left(Z_{n}, \ldots, Z_{n}^{k}\right)$, normalized so that $\left\|Z_{n}(0)\right\|=1$, on which all the $L^{p, q}$ vanish for $p+q<k$, and such that all the components stay bounded : this sequence (sub-)converges to a jet $\xi$, that is realisable, and on which all the $L^{p, q}$ vanish.

Remark 5. We want to finish with an open question related to the base point dependency of the type: in a complex case (see [5]), we know that the finite regular type is not an open condition ; but, if we consider all curves (even singular), this condition become open. Is it true for a almost complex structure and for pseudoholomorphic curves? We think that the answer is positive. 


\section{REFERENCES}

[1] T. Bloom. : On the contact between complex manifolds and real hyper-surfaces in $\mathbb{C}^{3}$, Trans. Amer. Math. Soc 263 (1981), 515-529.

[2] T. Bloom-I. Graham. : A geometric characterization of type on real submanifolds of $\mathbb{C}^{n}, \mathrm{~J}$. Diff. Geometry 12 (1977), 171-182.

[3] D.Catlin. : Subelliptic estimates for the $\bar{\partial}$-Neumann problem on pseudoconvex domains, Ann. of Math. 126 (1987), 131-191.

[4] D.Catlin. : Estimates of invariant metrics on pseudoconvex domains of dimension two, Math. Z. 200 (1989), 429-466.

[5] J.-P. D'Angelo. : Real hypersurface, orders of contact, and applications, Ann. of Math. 115 (1982), 615-637.

[6] J.-P. D'Angelo. : Several complex variables and the geometry of real hypersurfaces, Studies in Adv. Math. CRC press, Boca Raton, Fl, (1993).

[7] R. Debalme. : Variétés hyperboliques presque-complexes, Thèse de doctorat de l'université de Lille, (2001).

[8] J. Kohn. : Boundary behavior of $\bar{\partial}$ on weakly pseudoconvexmanifolds of dimension two, J. Diff Geometry 6 (1972), 523-542.

[9] J. Mcneal. : Estimates on the Bergman kernels of convex domains, Adv. Math 109 (1994), 108-139.

[10] J.-C. Sikorav. : Some properties of holomorphic cueves in almost complex manifolds, Prog. Math. 117, 165-189, Birkhauser, Basel, (1994).

UMR 8524, Université Lille 1, 59655 Villeneuve d’Ascq Cedex - FRANCE

UMR 8524, Université Lille 1, 59655 Villeneuve D’Ascq Cedex - FRANCE 